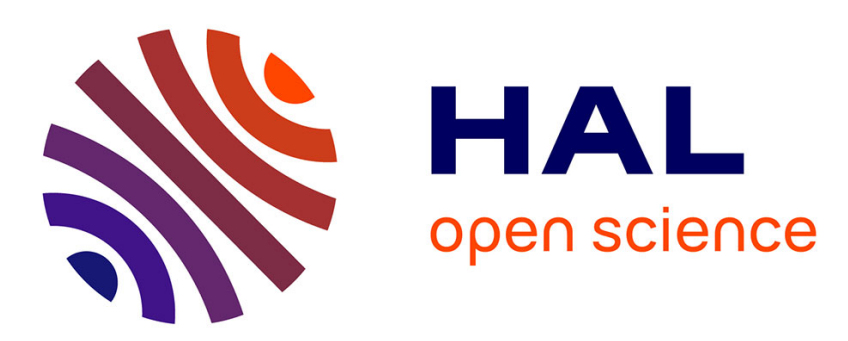

\title{
Area-time efficient hardware architecture for factoring integers with the elliptic curve method
}

\author{
Jan Pelzl, Martin Simka, Thorsten Kleinjung, Milos Drutarovsky, Viktor
}

Fischer, Christof Paar

\section{To cite this version:}

Jan Pelzl, Martin Simka, Thorsten Kleinjung, Milos Drutarovsky, Viktor Fischer, et al.. Area-time efficient hardware architecture for factoring integers with the elliptic curve method. Information Security, IEE Proceedings, 2005, 152 (1), pp.67-78. ujm-00289036

\section{HAL Id: ujm-00289036}

\section{https://hal-ujm.archives-ouvertes.fr/ujm-00289036}

Submitted on 19 Jun 2008

HAL is a multi-disciplinary open access archive for the deposit and dissemination of scientific research documents, whether they are published or not. The documents may come from teaching and research institutions in France or abroad, or from public or private research centers.
L'archive ouverte pluridisciplinaire HAL, est destinée au dépôt et à la diffusion de documents scientifiques de niveau recherche, publiés ou non, émanant des établissements d'enseignement et de recherche français ou étrangers, des laboratoires publics ou privés. 


\title{
Area-Time Efficient Hardware Architecture \\ for Factoring Integers with the Elliptic Curve Method
}

\author{
Jan Pelzl ${ }^{1}$, Martin Šimka², Thorsten Kleinjung ${ }^{3}$, \\ Miloš Drutarovský², Viktor Fischer ${ }^{4}$, Christof Paar ${ }^{1}$ \\ ${ }^{1}$ Horst Görtz Institute for IT Security, Ruhr University Bochum, Germany \\ \{pelzl, cpaar\}@crypto.rub.de \\ ${ }^{2}$ Department of Electronics and Multimedia Communications, Technical University of Košice, \\ Park Komenského 13, 04120 Košice, Slovak Republic \\ \{Martin.Simka, Milos.Drutarovsky\}@tuke.sk \\ ${ }^{3}$ University of Bonn, Department of Mathematics, Beringstraße 1, D-53115 Bonn, Germany \\ thor@math.uni-bonn.de \\ ${ }^{4}$ Laboratoire Traitement du Signal et Instrumentation, Unité Mixte de Recherche CNRS 5516, \\ Université Jean Monnet, 10, rue Barrouin, 42000 Saint-Etienne, France \\ fischer@univ-st-etienne.fr
}

\begin{abstract}
Since the introduction of public key cryptography, the problem of factoring large composites is of increased interest. The security of the most popular asymmetric cryptographic scheme RSA depends on the hardness of factoring large numbers. The best known method for factoring large integers is the General Number Field Sieve (GNFS). One important step within the GNFS is the factorization of mid-size numbers for smoothness testing, an efficient algorithm for which is the Elliptic Curve Method (ECM). Since the smoothness testing is also suitable for parallelization, it is promising to improve ECM via special-purpose hardware. We show that massive parallel and cost efficient ECM hardware engines can improve the cost-time product of the RSA moduli factorization via the GNFS considerably.
\end{abstract}


The computation of ECM is a classical example for an algorithm that can be significantly accelerated through special-purpose hardware. In this work, we thoroughly analyze the prerequisites for an area-time efficient hardware architecture for ECM. We present an implementation of ECM to factor numbers up to 200 bits, which is also scalable to other bit lengths. ECM is realized as a software-hardware co-design on an FPGA and an embedded microcontroller (system-on-chip). Furthermore, we provide estimates for state-of-the-art CMOS implementation of the design and for the application of massive parallel ECM engines to the GNFS. This appears to be the first publication of a realized hardware implementation of ECM, and the first description of GNFS acceleration through hardware-based ECM.

Keywords: factorization, elliptic curve method, system-on-chip, field programmable gate array.

\section{Introduction}

Since the ancient world, factoring integers has been investigated painstakingly by many mathematicians. With the dawn of asymmetric cryptography in the mid 1970s the problem of factoring large composites has again evoked increased mathematical interest. These days, the by far most popular asymmetric cryptosystem is RSA which was developed by Ronald Rivest, Adi Shamir and Leonard Adleman in 1977 [1]. The security of the RSA cryptosystem relies on the difficulty of factoring large numbers. Hence, the development of a fast factorization method could allow for cryptanalysis of RSA messages and signatures. However, till now the problem of factorization has remained hard.

Several efficient algorithms for factoring integers have been proposed. Each algorithm is appropriate for a different situation. For instance, the Elliptic Curve Method (ECM, see [2]) allows for efficient factoring of medium sized numbers with relatively small factors. The Generalized Number Field Sieve (GNFS, see [3]) is best for factoring numbers with large factors and, hence, can be used for attacking the RSA cryptosystem. However, as an intermediate step, the GNFS requires a method to efficiently factor lots of smaller numbers (factorization of the rests). An appropriate choice for this task is MPQS (Multiple Polynomial Quadratic Sieve) or ECM.

The current world record in factoring a random RSA modulus is 576 bits and was achieved with a complete software implementation of the GNFS in 2003 [4], using MPQS for the factoriza- 
tion of the rests. For larger moduli it will become crucial to use special hardware for factoring. Recently, some new hardware architectures for the sieving step in GNFS have been proposed (e.g., SHARK [5], TWIRL [6]). The efficiency of, e.g., SHARK (and possibly other innovative GNFS realizations) is directly related to efficient support units for smoothness testing within the architecture.

It appears that the use of ECM rather than MPQS is the better choice for this task, since the MPQS requires a larger silicon area and irregular operations. On the other hand, ECM is an almost ideal algorithm for dramatically improving the time-cost product through special purpose hardware. First, it performs a very high number of operations on a very small set of input data, and is, thus, not very I/O intensive. Second, it requires relatively little memory. Third, the operands needed for supporting GNFS are well beyond the width of current computer buses, arithmetic units, and registers, so that special purpose hardware can provide a much better fit. Lastly, it should be noted that the nature of the smoothness testing in the GNFS allows for a very high degree of parallelization. Hence, the key for efficient ECM hardware lies in fast arithmetic units. Such units for modular addition and multiplication have been studied thoroughly in the last few years, e.g., for the use in cryptographic devices using ECC (Elliptic Curve Cryptography), see, e.g., $[7,8]$. Therefore, we could exploit the well developed area of ECC architectures for our ECM design.

In this work, we present an efficient hardware implementation of ECM to factor numbers up to approximately 200 bits, which is also scalable to other bit lengths. We provide an elaborate specification of an area-time (AT) efficient ECM architecture, especially suited for hardware implementations. For proof-of-concept purpose, the ECM architecture has been realized as a softwarehardware co-design on an FPGA and an embedded microcontroller (system-on-chip). Timings of the real hardware for the operations of the ECM algorithm are presented. We also provide estimates for a state-of-the-art CMOS implementation of the design and for the application of massive parallel ECM engines to the GNFS. Our design has a good scalability to larger and smaller bit lengths. In some range, both the time and the silicon area depend linearly on the bit length. Such a design perfectly fits the needs of more recent proposals for hardware architectures for the GNFS (see, e.g., [5]) and can reduce the overall costs of a GNFS device considerably.

There are many possible improvements of the original ECM. Based on these improvements, 
we adapted the method to the very restrictive memory requirements of efficient hardware, thus, minimizing the AT product. The parameters used in our implementation are best suited to find factors of up to about 40 bits.

For the implementation, a highly efficient modular multiplication architecture described by Tenca and Koç (see [9]) is used and allows for reliable estimates for a future ASIC implementation. We propose to group about 1000 ECM units together on an ASIC and describe a controlling unit that synchronously feeds the units with programming steps, such that the ECM algorithm does not need to be stored in every single unit. In this way we keep the overall ASIC area small and still do not need much bandwidth for communication.

Section 3 introduces ECM and some optimizations relevant for our implementation. The choice of parameters and arithmetic algorithms, the design of the ECM unit and a possible parallelization are described in Section 4. The next two sections present our FPGA implementation, some estimates for a realization as ASIC and a case study of GNFS support with ECM hardware. The last section collects results and conclusions.

\section{Previous Work on ECM in Soft- and Hardware}

To our knowledge, ECM has never been implemented in hardware before. In the context of specialpurpose hardware for the GNFS, [10] mentions, that the construction of special ECM hardware might be promising for supporting the GNFS. However, till now we are not aware of any publication dealing with ECM hardware.

ECM is related to Elliptic Curve Cryptosystems (ECC), hence, hardware implementations of recent ECC using Montgomery coordinates can also be of interest. The advantage of the use of Montgomery form curves in cryptography is the inherit resistance against side channel attacks due to almost indistinguishable group operations. I.e., the elementary operations for addition and duplication of points are quite similar. A handicap of the Montgomery form is the fact that not every arbitrary curve can be transformed into Montgomery form. Hence, there is merely interest in implementing ECC based on Montgomery form curves.

A parallel software implementation of ECM on several workstations (PentiumII@350 MHz, Linux OS) is reported in [11]. The implementation uses fast network switches and has been programmed based on the Message-Passing Interface (MPI) standard. 
A well known free software implementation of the Elliptic Curve Method (ECM) to factor integers is available from [12] (GMP-ECM). The implementation is based on the GNU Multiple Precision Arithmetic Library (GMP). The original purpose of the project was to find a factor of 50 digits or more by ECM. By participation of several developers, GMP-ECM is an excellent resource for a state-of-the-art ECM software implementation, including many useful algorithms.

\section{$3 \quad$ Elliptic Curve Method}

The principles of ECM are based on Pollard's $(p-1)$-method ([13]). A short summary of Pollard's method can be found in Appendix A.1. In the following, we describe H. W. Lenstra's Elliptic Curve Method (ECM, see [2]).

\subsection{The Algorithm}

Let $N$ be an integer without small prime factors which is divisible by at least two different primes, one of them $q$. Such numbers appear after trial division and a quick prime power test.

Let $E / \mathbb{Q}$ be an elliptic curve with good reduction at all prime divisors of $N$ (this can be checked by calculating the gcd of $N$ and the discriminant of $E$ which very rarely yields a prime factor of $N)$ and a point $P \in E(\mathbb{Q})$. Let $E(\mathbb{Q}) \rightarrow E\left(\mathbb{F}_{q}\right), Q \mapsto \bar{Q}$ be the reduction modulo $q$. If the order $o$ of $\bar{P} \in E\left(\mathbb{F}_{q}\right)$ satisfies certain smoothness conditions described below, we can discover the factor $q$ of $N$ as follows: In the first phase of ECM, we calculate $Q=k P$ where $k$ is a product of primes

$p \leq B_{1}$ and prime powers $p^{e} \leq B_{2}$ with appropriate chosen smoothness bounds $B_{1}, B_{2}$. The second phase of ECM checks for each prime $B_{1}<p \leq B_{2}$ whether $p Q$ reduces to the neutral element in $E\left(\mathbb{F}_{q}\right)$. Algorithm (1) summarizes all necessary steps for both phases of ECM. Phase 2 can be done efficiently, e.g., using the Weierstraß form and projective coordinates $p Q=\left(x_{p Q}: y_{p Q}: z_{p Q}\right)$ by testing whether $\operatorname{gcd}\left(z_{p Q}, N\right)$ is bigger than 1 . Note that we can avoid all gcd computations but one at the expense of one modular multiplication per ged by accumulating the numbers to be checked in a product modulo $N$ and performing one final gcd.

If using only one single curve, the properties of ECM are related to those of Pollard's $(p-1)$ method. The advantage of ECM lies in the possibility of choosing a different curve after each trial to increase the probability of finding factors of $N$. 


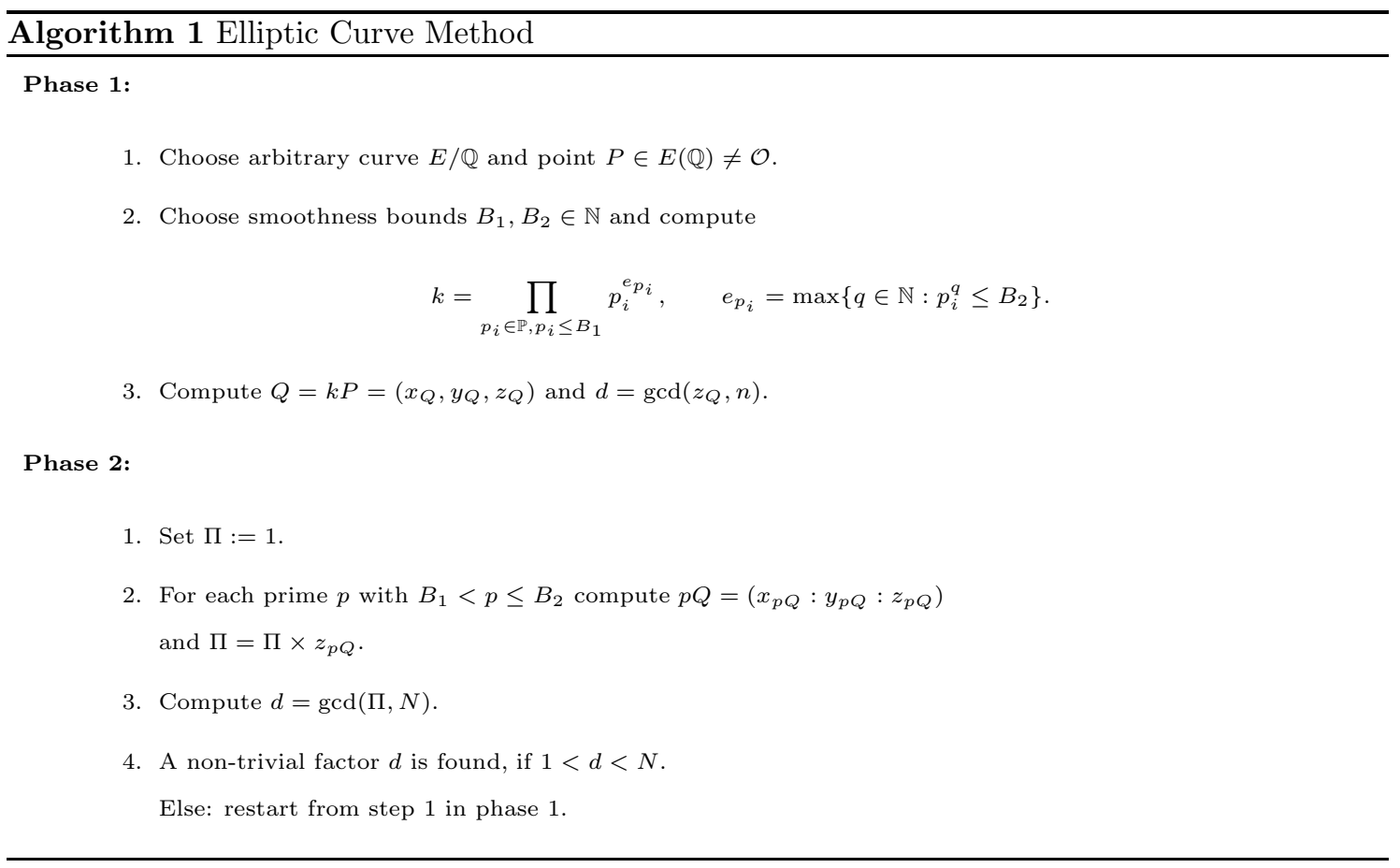

All calculations are done modulo $N$. If the final ged of the product $\Pi$ and $N$ satisfies $1<\operatorname{gcd}(\Pi, N)<N$, a factor is found. The parameters $B_{1}$ and $B_{2}$ control the probability of finding a divisor $q$. More precisely, if $o$ factors into a product of coprime prime powers (each $\left.\leq B_{1}\right)$ and at most one additional prime between $B_{1}$ and $B_{2}$, the prime factor $q$ is discovered.

The procedure will be repeated for other elliptic curves and starting points. To generate them one commences with the starting point $P$ and constructs an elliptic curve such that $P$ lies on it.

It is possible that more than one or even all prime divisors of $N$ are discovered simultaneously. This happens rarely for reasonable parameter choices and can be ignored by proceeding to the next elliptic curve.

The running time of ECM is given by

$$
T(p)=e^{\sqrt{2 \log p \log \log p}(1+o(1))},
$$

thus, it depends on the size of the factors to be found and not on the size of $N[14]$. 


\subsection{The Elliptic Curves}

Apart from the Weierstraß form there are various other forms for the elliptic curves. We use Montgomery's form (1) and compute in the set $S=E(\mathbb{Z} / N \mathbb{Z}) /\{ \pm 1\}$ only using the $x$ - and $z$ coordinates.

$$
B y^{2} z=x^{3}+A x^{2} z+x z^{2}
$$

It was suggested in [15] by Montgomery. Curves of this form always have an order divisible by 4. I.e., not every curve can be transformed to the Montgomery form. In our case, the curves can be chosen in such way that they have an order divisible by 12 . Appendix A.2 describes the construction of such curves.

The residue class of $\overline{P+Q}$ in this set can be computed from $\bar{P}, \bar{Q}$ and $\overline{P-Q}$ using 6 multiplications (2). A duplication, i. e. $\overline{2 P}$, can be computed from $\bar{P}$ using 5 multiplications (3). Since we are only interested in checking whether we obtain the point at infinity for some prime divisor of $N$ computing in $S$ is no restriction. In the following we will not distinguish between $E(\mathbb{Z} / N \mathbb{Z})$ and $S$, and pretend to do all computations in $E(\mathbb{Z} / N \mathbb{Z})$.

\section{Addition:}

$$
\begin{aligned}
x_{P+Q} & \equiv z_{P-Q}\left[\left(x_{P}-z_{P}\right)\left(x_{Q}+z_{Q}\right)+\left(x_{P}+z_{P}\right)\left(x_{Q}-z_{Q}\right)\right]^{2} \quad(\bmod N) \\
z_{P+Q} & \equiv x_{P-Q}\left[\left(x_{P}-z_{P}\right)\left(x_{Q}+z_{Q}\right)-\left(x_{P}+z_{P}\right)\left(x_{Q}-z_{Q}\right)\right]^{2} \quad(\bmod N)
\end{aligned}
$$

\section{Duplication:}

$$
\begin{aligned}
4 x_{P} z_{P} & \equiv\left(x_{P}+z_{P}\right)^{2}-\left(x_{P}-z_{P}\right)^{2}(\bmod N) \\
x_{2 P} & \equiv\left(x_{P}+z_{P}\right)^{2}\left(x_{P}-z_{P}\right)^{2}(\bmod N) \\
z_{2 P} & \equiv 4 x_{P} z_{P}\left[\left(x_{P}-z_{P}\right)^{2}+4 x_{P} z_{P}(A+2) / 4\right] \quad(\bmod N)
\end{aligned}
$$

\subsection{The First Phase}

If the triple $(P, n P,(n+1) P)$ is given we can compute $(P, 2 n P,(2 n+1) P)$ or $(P,(2 n+1) P,(2 n+2) P)$ by one addition and one duplication in Montgomery's form. Thus $Q=k P$ can be calculated using $\left[\log _{2} k\right]$ additions and duplications according to Algorithm 2, amounting to $11\left[\log _{2} k\right]$ multiplica- 
tions. In the case $z_{P}=1$ we can reduce this to $10\left[\log _{2} k\right]$ multiplications.

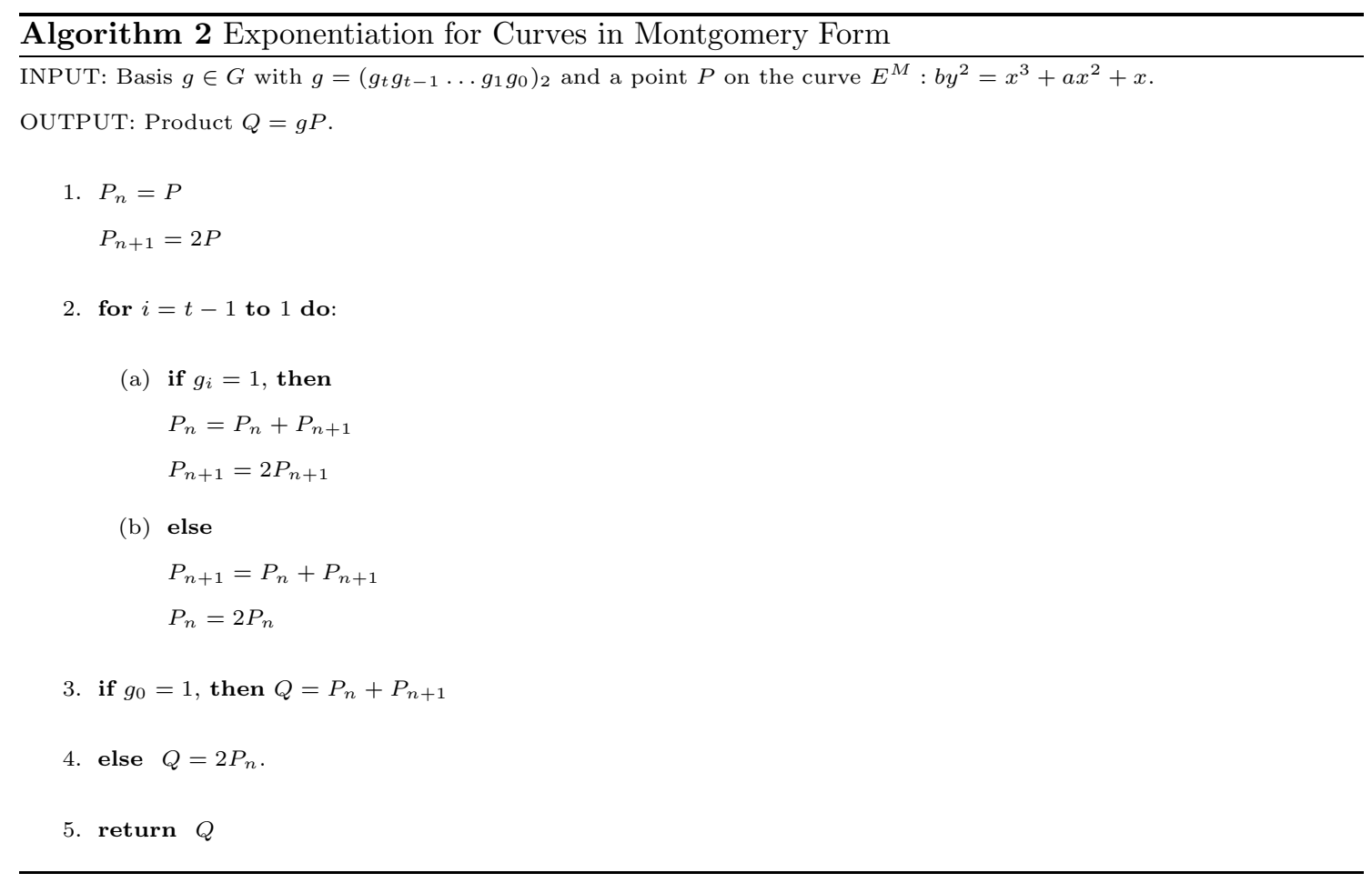

By handling each prime factor of $k$ separately and using optimal addition chains the number of multiplications can be decreased to roughly $9.3\left[\log _{2} k\right]$ (see [15]). The addition chains can be precalculated.

\subsection{The Second Phase}

The standard way to calculate the points $p Q$ for all primes $B_{1}<p \leq B_{2}$ is to precompute a (small) table of multiples $k Q$ where $k$ runs through the differences of consecutive primes in the interval $\left.] B_{1}, B_{2}\right]$. Then, $p_{0} Q$ is computed with $p_{0}$ being the smallest prime in that interval and the corresponding table entries are added successively to obtain $p Q$ for the next prime $p$. Two major improvements have been proposed for ECM $([16,15])$. Using Montgomery's form, the procedure is difficult to implement but can be improved as follows.

The improved standard continuation uses a parameter $2<D<B_{1}$. First, a table $T$ of multiples $k Q$ of $Q$ for all $1 \leq k<\frac{D}{2}, \operatorname{gcd}(k, D)=1$ is calculated. Each prime $B_{1}<p \leq B_{2}$ can be written as $m D \pm k$ with $k Q \in T$. Now, with Lemma 1 (Appendix A.3), $\operatorname{gcd}\left(z_{p Q}, N\right)>1$ if and only if $\operatorname{gcd}\left(x_{m D Q} z_{k Q}-x_{k Q} z_{m D Q}, N\right)>1$. Thus, we calculate the sequence $m D Q$ (which can easily be 
done in Montgomery's form) and accumulate the product of all $x_{m D Q} z_{k Q}-x_{k Q} z_{m D Q}$ for which $m D-k$ or $m D+k$ is prime.

The memory requirements for the improved standard continuation are $\frac{\varphi(D)}{2}$ points for the table $T$ and the points $D Q,(m-1) D Q, m D Q$ for computing the sequence, altogether $\varphi(D)+6$ numbers. The computational costs consist of the generation of $T$ and the calculation of $m D Q$ which amounts to at most $\frac{D}{4}+\frac{B_{2}}{D}+7$ elliptic curve operations (mostly additions) and at most $3\left(\pi\left(B_{2}\right)-\pi\left(B_{1}\right)\right)$ modular multiplications, $\pi(x)$ being the number of primes up to $x$. The last term can be lowered if $D$ contains many small prime factors since this will increase the number of pairs $(m, k)$ for which both $m D-k$ and $m D+k$ are prime. Neglecting space considerations a good choice for $D$ is a number around $\sqrt{B_{2}}$ which is divisible by many small primes.

\section{Methodology}

We will now discuss the parameterization of ECM, optimized for our purposes. Finally, the design of the ECM unit is described.

\subsection{Parameterization of the ECM Algorithm}

Our implementation focusses on the factorization of numbers up to 200 bits with factors of up to around 40 bits. Thus, "good" parameters for $B_{1}, B_{2}$, and $D$ have to be found, yielding a high probability of success and a relatively small running time and area consumption. With the running time depending on the size of the (unknown) factors to be found, optimal parameters cannot be known beforehand. Hence, good parameters can be found by experiments with different prime bounds.

Deduced from software experiments, we choose $B_{1}=960$ and $B_{2}=57.000$ as prime bounds. The value of $k$ has 1375 bits, hence, assuming the binary method (Algorithm 2), 1374 point additions and point duplications for the execution of phase 1 are required. Due to the use of Montgomery coordinates, the coordinate $z_{P}$ of the starting point $P$ can be set to 1 , thus, addition takes only 5 multiplications instead of 6 . The improved phase 1 (with optimal addition chains) has to use the general case, where $z_{P} \neq 1$. For the sake of simplicity and a preferably simple control logic, we choose the binary method for the time being. For the chosen parameters, the computa- 
tional complexity of phase 1 is 13740 modular multiplications and squarings ${ }^{1}$. (With optimized addition chains this number can be reduced to approximately 12000 modular multiplications and squarings.)

According to Equation (3), duplicating a point $2 P_{A}=P_{C}$ involves the input values $x_{A}, z_{A}, A_{24}$ and $N$, where $A_{24}=(A+2) / 4$ is computed from the curve parameter $A$ (see Equation (1)) in advance and should be stored in a fixed register. A point addition $P_{C}=P_{A}+P_{B}$ handles the input values $x_{A}, z_{A}, x_{B}, z_{B}, x_{A-B}, z_{A-B}$ and $N$ (see Equation 2). Notice that during phase 1 the values $N, A_{24}, x_{A-B}$ and $z_{A-B}$ do not change. Furthermore, $z_{A-B}=z_{1}$ can be chosen to be 1 . Thus, no register is required for $z_{A-B}$. The output values $x_{C}$ and $z_{C}$ can be written to certain input registers to save memory. If we assume that the ECM unit does not execute addition and duplication in parallel, at most 7 registers for the values in $\mathbb{Z} / n \mathbb{Z}$ are required for phase 1 . Additionally, we will require 4 temporary registers for intermediate values. Thus, a total of 11 registers is required for phase 1 .

For the prime bounds chosen, 5621 primes $p \in\left[B_{1}, B_{2}\right]$ have to be tested in phase 2 . With the prime bounds fixed, the computational complexity depends on the size of $D$. Hence, $D$ should consist of small primes in order to keep $\varphi(D)$ as small as possible. We consider the cases $D=6$, $D=30, D=60$ and $D=210$. The initial values can be computed by first computing $\hat{Q}=$ $D Q$, then $\frac{B_{1}}{D} \hat{Q}$ with the binary method, yielding automatically $\left(\frac{B_{1}}{D}-1\right) \hat{Q}$. The total number of modular multiplications is determined by the number of point additions, point duplications and multiplications for the product $\Pi$. Table 1 displays the computational complexity and the number of registers required additionally for phase 2 . For the numbers in the table, we assume the use of Algorithm 2 for computing the initial values. E.g., in the case $D=30$, the cost for the computation of $D Q,\left(\frac{B_{1}}{D}-1\right) D Q$, and $\frac{B_{1}}{D} D Q$ is as much as 8 point additions and 8 point duplications. For the same $D$, the computation of the table involves 5 point additions and 2 point duplications, yielding to a total of 13590 modular multiplications.

Remark: for the case $D=210$, we start with $B_{1}=1050$ in order to assure that $D$ and $B_{1}$ share the same prime factors.

For phase 2 we choose $D=30$ to obtain a minimal AT product of the design. Since $\varphi(D)=8$ is small, only 8 additional registers are required to store all coordinates in a table. Unlike in phase 1 ,

\footnotetext{
${ }^{1}$ In this contribution, squarings and multiplications are considered to have an identical complexity since the hardware will compute a squaring with the multiplication circuit.
} 
Table 1: Computational Complexity and Memory Requirements for Phase 2 depending on $D$

\begin{tabular}{|c||c|c|c|c|c|}
\hline \multicolumn{1}{|c||}{} & \multicolumn{3}{c|}{ number of modular multiplications for } & number \\
$D$ & point additions & point duplications & product $\Pi$ & total & of regs. \\
\hline \hline 6 & $(9+0+9340) \times 6=56094$ & $(9+0) \times 5=45$ & 14625 & 70764 & 4 \\
\hline 30 & $(8+5+1868) \times 6=11286$ & $(8+2) \times 5=50$ & 13590 & 24926 & 10 \\
\hline 60 & $(8+9+934) \times 6=5706$ & $(8+2) \times 5=50$ & 13629 & 19385 & 18 \\
\hline 210 & $(9+28+266) \times 6=1818$ & $(9+5) \times 5=70$ & 13038 & 14926 & 50 \\
\hline
\end{tabular}

we have to consider the general case for point addition where $z_{A-B} \neq 1$. Hence, an additional register for this quantity is needed. For the product $\Pi$ of all $x_{A} \times z_{B}-z_{A} \times x_{B}$, one more register is necessary. The temporary registers from phase 1 suffice to store the intermediate results $x_{A} \times z_{B}$, $z_{A} \times x_{B}$ and $x_{A} \times z_{B}-z_{A} \times x_{B}$. Hence, additional 10 registers for phase 2 yield to a total of 21 required registers for both phases. The computational complexity of phase 2 is 1881 point additions 10 point duplications. Together with the 13590 modular multiplications for computing the product $\Pi, 24926$ modular multiplications and squarings are required.

For a high probability of success $(>90 \%)$ for the parameters given, software experiments suggest to run ECM on approximately 20 different curves.

\subsection{Design of the ECM Hardware}

The ECM unit mainly consists of three parts: the ALU (Arithmetic Logic Unit), the memory part (register) and an internal control logic (see Figure 1). Each unit has a very low communication overhead since all results are stored inside the unit during computation. Before the actual computation starts, all required initial values $\left(x_{P}, N, A_{24}\right)$ are assigned to memory cells of the unit. Only at the very end when the product $\Pi$ has been computed, the result is read from the unit's memory. Commands are generated and timed by a central control logic outside the ECM unit(s).

\subsubsection{Central Control Logic}

The central control logic is connected to each ECM unit via a control bus (ctrl). It coordinates the data exchange with the unit before and after computation and starts each computation in the unit 
Figure 1: Overview of one ECM Unit

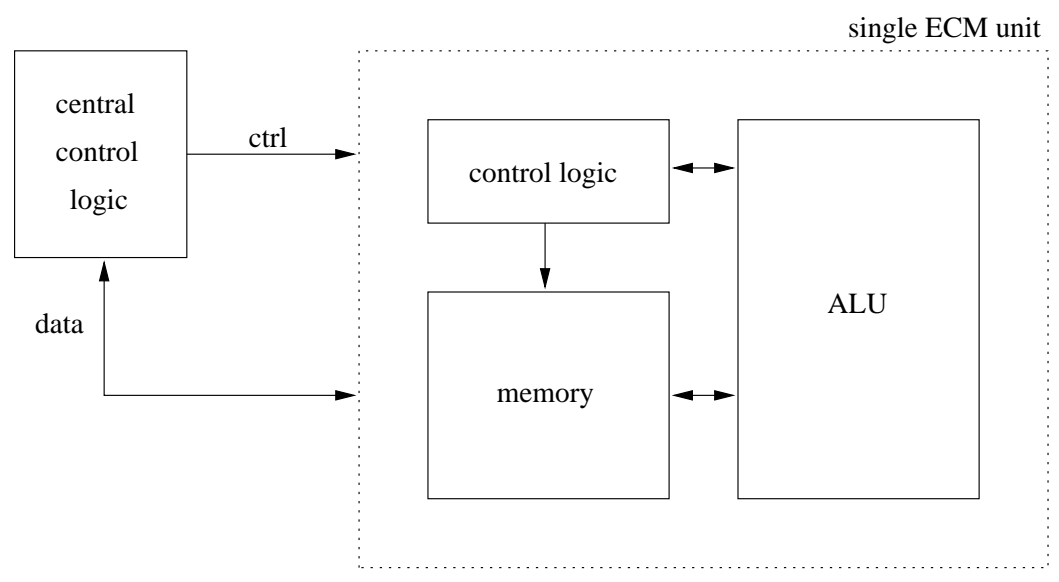

by a special set of commands. The commands contain an instruction for the next computation to be performed (i.e., add, subtract, multiply, double), including the in- and output registers to be used (R1-R21). The start of an operation is invoked by setting the start-bit to '1'.

The control bus has to offer the possibility to specify which input register(s) and which output register is active. Only certain combinations of in- and output registers occur, offering the possibility to reduce the complexity of the logic and the width of the control bus by compressing the necessary information. For simplicity and clarity, we skipped the further optimization of the commands. Instead, we use a clearly understandable structure for the commands. A command consists of 16 bit which are assigned as follows (LSB is left):

\begin{tabular}{c|c|c|c|c} 
start & operation & input 1 & input 2 & output \\
\hline $\mathrm{X}$ & $\mathrm{XX}$ & $\mathrm{Xxxx}$ & $\mathrm{Xxxx}$ & $\mathrm{Xxxxx}$
\end{tabular}

If several ECM units work in parallel, only one central control logic is needed. All commands are sent in parallel to all units. Only in the beginning and in the end, the unit's memory cells have to be written and read out separately. Once the computations in all units finished, an LSB of the central status register is set to '0' to indicate the units' availability for further commands.

\subsubsection{Internal Control Logic}

Each unit possesses an internal control logic in order to coordinate the data in- and output from and to the registers, respectively. Once a command with the corresponding start bit is set, the computation inside the unit is started. Once the computation is finished, a bit is set to ' 1 ' to 
indicate the unit's availability for further commands. The modular arithmetic is coordinated inside the ALU and communicated with the internal control logic.

\subsubsection{Memory}

The addresses specified above refer to relative addresses inside each unit since we want to address the same register in multiple units in parallel. For reading from/ writing to a single register in a specific unit, the unit needs to be addressed separately. In combination with a unique address for each unit, a register has a unique hardware address and can be addressed from outside the unit. This is imperative since the central control logic writes data to these registers before phase 1 starts and it reads data from one of the registers after phase 2 has been finished. Each register can contain $n$ bits and is organized in $e=\left\lceil\frac{n+1}{w}\right\rceil$ words of size $w$ (see Figure 2). Memory access is performed word wise. Reasonable values for $w$ are $w=4,8,16,32$ but are, though, not mandatory.

Figure 2: Memory Management of the ECM Unit

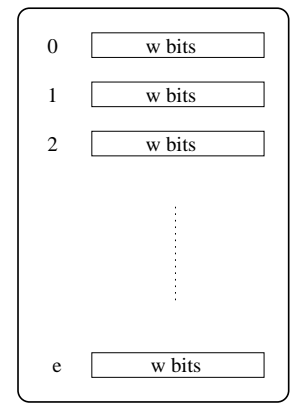

$P_{1}$

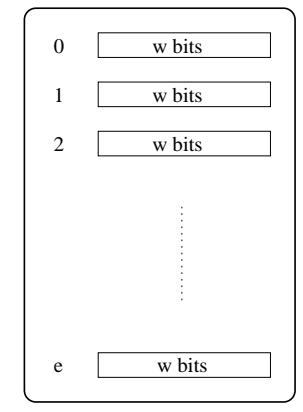

$P_{21}$

\subsubsection{Arithmetic Logic Unit (ALU)}

The ALU performs the arithmetic modulo $2 N$, i.e., modular multiplication, modular squaring, modular addition and subtraction. Possible benefit from using multiple ALUs in one ECM unit is discussed in Appendix A.4.

Objectives for the choice of implemented algorithms are mentioned in Subsection 4.3. 


\subsection{Choice of the Arithmetic Algorithms}

The main purpose of the design is to synthesize an area-time efficient implementation of ECM. Hence, all algorithms are chosen to allow for low area and relatively high speed. Low area consumption can be achieved by structures, which allow for a certain degree of serialization and, hence, do not require much memory. For ECM, we have chosen a set of algorithms which seem to be very well suited for our purpose. In the following, we briefly describe the algorithms for modular addition, subtraction, and multiplication to be implemented for the ALU. Squaring is done with the multiplication circuit since a separate hardware circuit for squaring would increase the overall AT product. Similarly, subtraction can be computed with a slightly changed adder circuit.

\subsubsection{Computing with Montgomery Residues}

It is well known that the Montgomery multiplication ([17]) is an efficient method for modular multiplication. Montgomery's algorithm replaces divisions by simple shift operations and, thus, is very efficient in hard- and software. The method is based on a representation of the residue class modulo the modulus $N$. The corresponding $N$-residue of an integer $x$ is defined as

$$
x^{\prime}=x \times r \bmod N
$$

where $r=2^{n}$ with $2^{n-1}<N<2^{n}$ such that $\operatorname{gcd}(r, N)=1$ (which is always true for $N$ odd). Since it is costly to switch always between integers and their $N$-residue and vice versa, we will perform all computations in the residue class.

\subsubsection{Modular Multiplication}

An efficient Montgomery multiplier, highly suitable for our design is described in [9]. The presented multiplier architecture allows for word wise multiplication and is scalable regarding operand size, word size, and pipelining depth. The internal word additions are performed by simple adders. Figure 3 shows the architectures of one stage. While in [9] a structure with carry-save adders and redundant representation of operands has been implemented, we have chosen a configuration with carry-propagate adders and non-redundant representation that makes possible more effective implementation especially when target platform supports fast carry chain logic. A detailed analysis and comparison of both structures can be found in [18]. 
Figure 3: Multiplier Stage with Carry-Propagate Adders and Non-redundant Representation

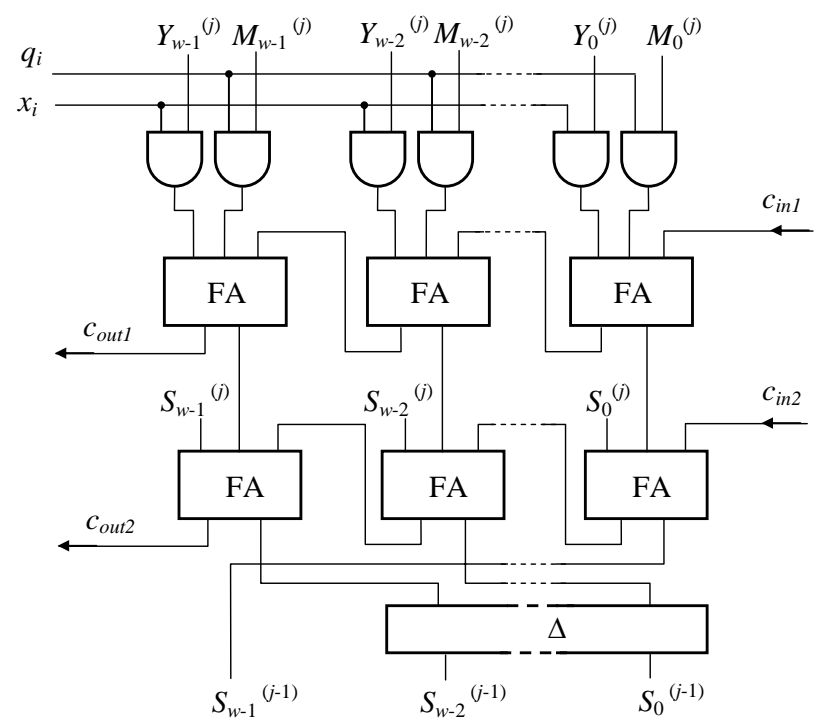

The depicted hardware performs a slightly modified Multiple Word Radix-2 Montgomery Multiplication (Algorithm 3). Instead of more expensive word-wise addition in step (a) we have used only bit operations. In Algorithm 3, word and bit vectors are represented as:

$$
\begin{aligned}
& N=\left(0, N^{(e-1)}, \ldots, N^{(1)}, N^{(0)}\right) ; Y=\left(0, Y^{(e-1)}, \ldots, Y^{(1)}, Y^{(0)}\right) ; S=\left(0, S^{(e-1)}, \ldots, S^{(1)}, S^{(0)}\right) \\
& X=\left(x_{n-1}, \ldots, x_{1}, x_{0}\right), \text { where words are marked with superscripts and bits are marked with }
\end{aligned}
$$
subscripts.

The final reduction step of originally proposed Montgomery multiplication can be omitted when the following condition is fulfilled:

$$
4 M<2^{n}
$$

With bounded input values $X, Y<2 M$, the output value is also bounded $(S<2 M)$.

According to [9], the number of clock cycles per multiplication is given by Equation (4):

$$
T_{m u l}=\left\lceil\frac{n}{p}\right\rceil \times(e+1)+2 p
$$

A minimal $A T$ product of the sole multiplier can be achieved with a word width of 8 bits and a pipelining depth of $1(w=8, p=1$, see [9]). However, for our ECM architecture, the AT product does not only depend on the $A T$ product of the multiplier. In fact, the multiplier only takes a comparably small part of the overall area. On the other hand, the overall speed relies primarily on the speed of the multiplier. Thus, we choose a pipelining depth of $p=2$ for word width $w=32$ bits, in order to achieve a higher speed. 


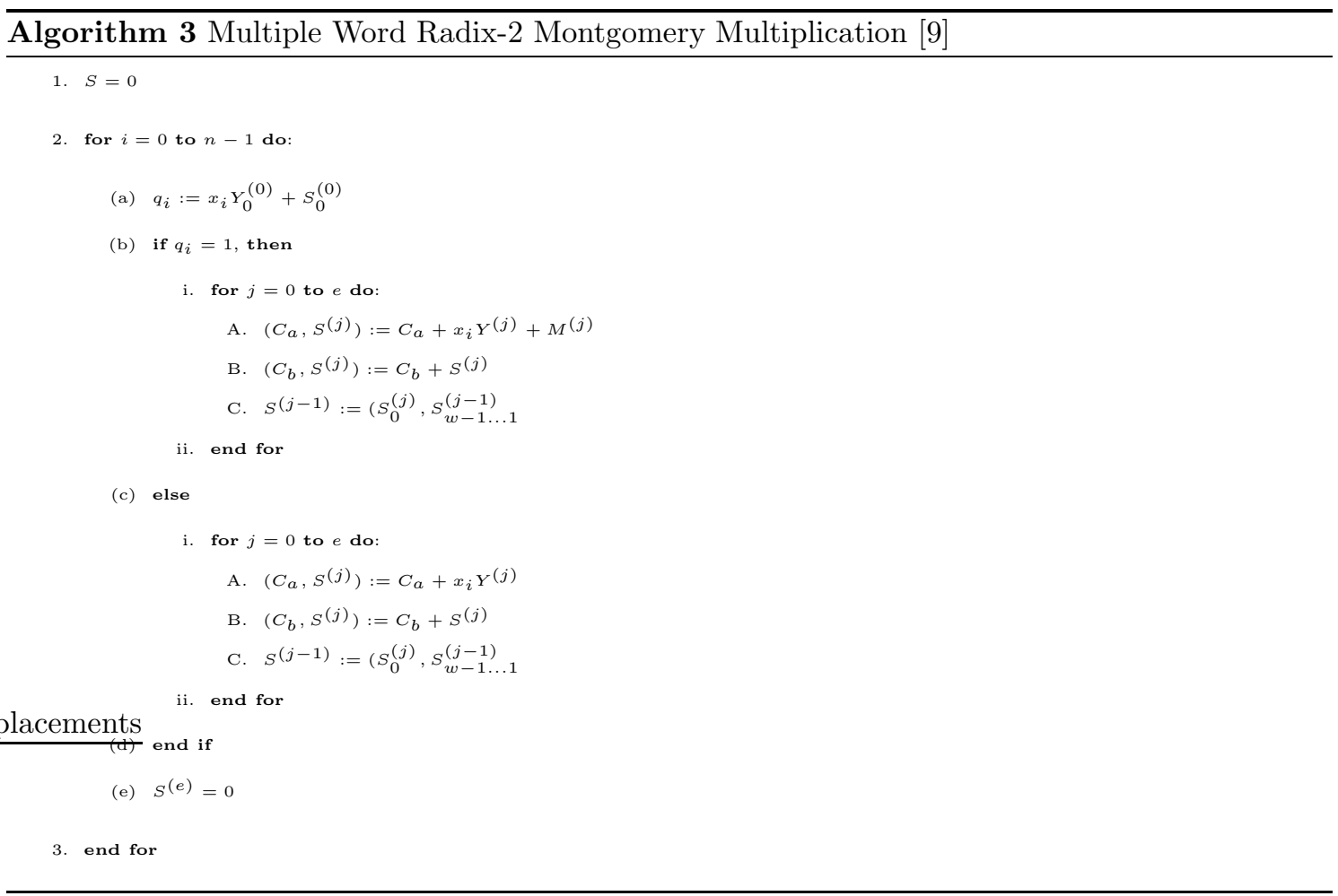

\subsubsection{Modular Addition and Subtraction}

Addition and subtraction is implemented as one circuit. As with the multiplication circuit, the operations are done word wise and the word size and number of words can be chosen arbitrary. Since the same memory is used for input and output operands, we choose the same word size as for the multiplier. The subtraction relies on the same hardware, only one input bit has to be changed $(s u b=1)$ in order to compute a subtraction rather than an addition (see Figure 4 , FA denotes a full adder). All operations are done modulo $2 N$.

Figure 4: Addition and Subtraction Circuit
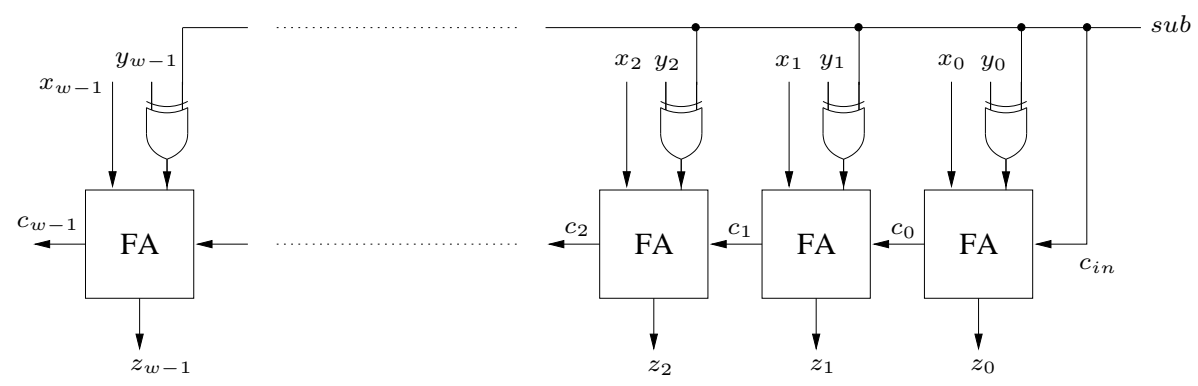
The actual computation is done in a word wise manner using a word size of $w=32$. Algorithms 4 and 5 show the elementary steps of a modular addition and subtraction, respectively.

If $|x+y|<2 N$ a reduction can be applied by simple subtraction of twice the modulus $2 N$. Hence, following algorithm is used for the modular addition:

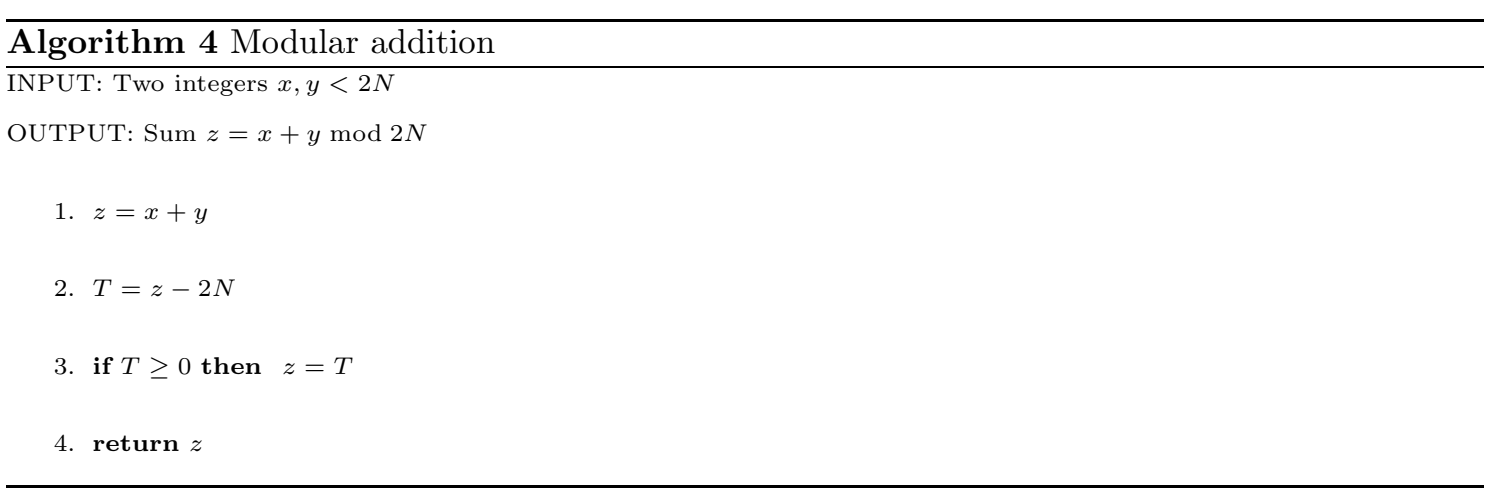

$z$ contains the result and $T$ is a (temporary) register. A comparison $z<2 N$ takes the same amount of time than a subtraction $T=z-2 N$. Thus, we compute the subtraction in all cases and decide by the sign of the values, which one to take as the result $(z$ or $T)$. If $T$ is the correct result, the content of $T$ has to be copied to the register $z$.

For a modular addition, we need at most

$$
T_{a d d}=3 \times(e+1)
$$

clock cycles, where $e$ is the number of words (for implemented non-redundant form of operands $\left.e=\left\lceil\frac{n+1}{w}\right\rceil\right)$. On average, we would only have to reduce every second time. However, since the control of phase 1 and phase 2 is parallelized for many units, we have to assume the worst case running time which is given by Equation (5).

The subtraction $x-y$ can be accomplished by the addition of $x$ with the bitwise complement of $y$ and 1 . The addition of 1 is simply achieved by setting the first carry bit to one $(c=1)($ Step 1$)$. Since the result can be negative, a final verification is required. If necessary, the modulus has to be added. Following algorithms describes the modular subtraction:

In step 1 , both memory cells $z$ and $T$ obtain the same value, which can be done in hardware in parallel at the same time without any additional overhead. After the computation of the difference, one can check for the correctness of the result. 


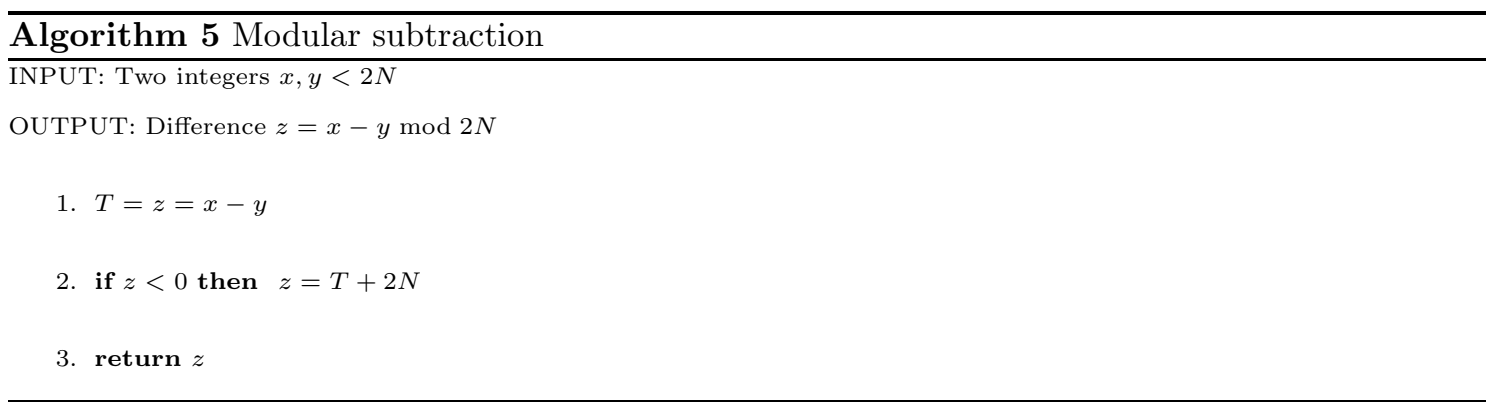

Hence, subtraction can be performed more efficiently than addition and requires in the worst case

$$
T_{s u b}=2 \times(e+1)
$$

clock cycles.

\subsection{Parallel ECM}

ECM can be perfectly parallelized by using different curves in parallel since the computations of each unit are completely independent. For the control of more than one ECM unit, it is essential to know that both phases, phase 1 and phase 2, are controlled completely identical, independent of the composite to be factored. Figure 5 shows the control of several ECM units in parallel.

Figure 5: Parallel ECM

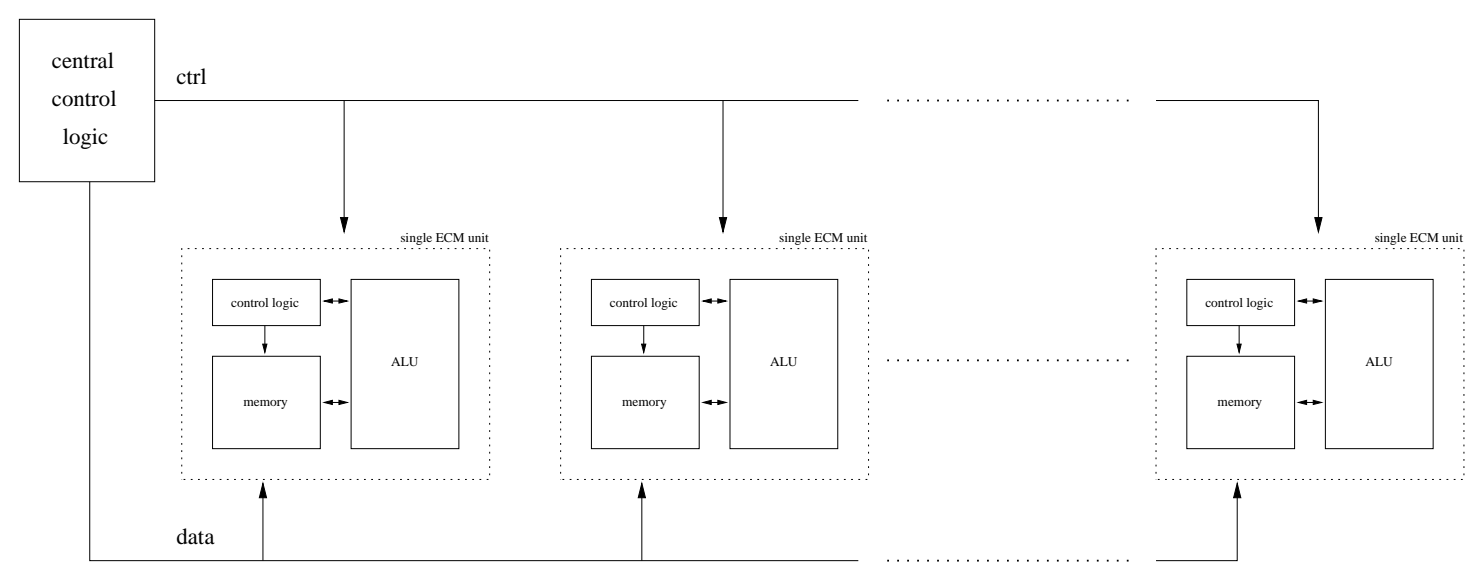

Solely the curve parameter and eventually the modulus of the units and, hence, the coordinates of the initial point differ. Thus, all units have to be initialized differently which is done by simply writing the values into the corresponding memory locations sequentially. During the execution of 
both phases, exactly the same commands can be sent to all units in parallel. Since running time of multiplication/squaring is constant (does not rely on input values) and for addition/subtraction differs at most in $2(e+1)$ clock cycles, all units can execute the same command at exactly the same time. After phase 2, the results are read from the units one after another. The required time for this data $\mathrm{I} / \mathrm{O}$ is neglectable for one ECM unit since the computation time of both phases dominates. For several units in parallel, the computation time does not change, but the time for data I/O scales linearly with the number of units. Hence, not too many units should be controlled by one single logic. For massively parallel ECM in hardware, the ECM units can be segmented into clusters, each with an own control unit.

\section{$5 \quad$ Implementation}

This section presents the actual hardware implementation done on a system-on-chip (FPGA and embedded microprocessor). This first hardware implementation of ECM is designed as a proof-ofconcept. Remark, that all timings are obtained by using real hardware, not only simulation.

\subsection{Hardware Platform}

The ECM implementation is realized as a hybrid design. It consists of an ECM unit implemented on an FPGA (Xilinx Virtex2000E-6) and a control logic implemented in software on an embedded microcontroller (ARM7TDMI, 25MHz). For more information on the hardware platform used, the interested reader is referred to [19]. The ECM unit is coded in VHDL and was synthesized for a Xilinx FPGA (Virtex2000E-6, see [20]). For the actual VHDL implementation, memory cells have been realized with the FPGA's internal block RAM. For simulation, ModelSim was used, synthesis and place\&route was done using FPGA Advantage's RTL tools.

The unit, as implemented, listens for commands which are written to control register accessible by the FPGA. Required point coordinates and curve parameters are loaded into the unit before the first command is decoded. For this purpose, these memory cells are accessible from the outside by a unique address. Internal registers, which are only used as temporary registers during the computation are not accessible from the outside.

The control of the unit is done by the microcontroller present on the board and controls the data transfer from and to the units, and issues the commands for all steps in phase 1 and phase 2 . 
For code generation, debugging and compilation, the ARM Developer Suite 1.2 was used. For details on the ARM microprocessor, see [21]. At a later stage, a soft coded processor core (in VHDL) could be used instead an ARM microprocessor.

The actual design was done for $n=198$ bit composites. The parameters for the multiplier are $p=2$ and $w=32$. Scaling the design to bit lengths from 100 to 300 bits can be easily accomplished. In this case, the $A T$ product will de-/ increase according to the size of $n^{2}$.

\subsection{Results}

After the synthesis and place and route, the binary image was loaded onto the FPGA and clocked with a frequency of $25 \mathrm{MHz}$. Hence, the cycle length of the ALU performing the modular arithmetic is 40ns. Table 2 shows the timings of relevant operations of the implementation. The timings of both phases include the time required for data I/O. For the FPGA used, the actual design has a maximum clock frequency of approximately $38 \mathrm{MHz}$. Due to the limits of the board's clock generation capabilities, only $25 \mathrm{MHz}$ are applied for our timing analysis.

Table 2: Running Times of the ECM Implementation (198 bits modulus), $p=2, w=32$ (Xilinx Virtex2000E-6 and ARM7TDMI, 25MHz)

\begin{tabular}{|c|c|}
\hline Operation & Time \\
\hline \hline modular addition & $2.16 \mu \mathrm{s}$ \\
modular subtraction & $2.00 \mu \mathrm{s}$ \\
modular multiplication & $64.5 \mu \mathrm{s}$ \\
modular squaring & $64.3 \mu \mathrm{s}$ \\
\hline \hline point addition (phase $\left.1, z_{Q}=1\right)$ & $334 \mu \mathrm{s}$ \\
point addition (phase 2) & $399 \mu \mathrm{s}$ \\
point duplication & $331 \mu \mathrm{s}$ \\
\hline \hline Phase 1 & $918 \mathrm{~ms}$ \\
Phase 2 (estimate) & $1760 \mathrm{~ms}$ \\
\hline
\end{tabular}


Although a squaring is computed with the multiplication circuit, the overhead is slightly lower yielding a mere $0.3 \%$ faster execution. Point addition in phase 1 is more efficient since it makes use of the fact, that the $z$ coordinate of the difference of points can be chosen to be 1 .

Remark, that the work on phase 2 is still in progress. Though the FPGA part of phase 2 has been completed, the complicated control logic has still to be optimized and partially revised. Parts of phase 2 are already running on the platform, some parts are in progress. Hence, we provide an estimate of the running time based on the running time of the timings given in Table 2. Based on experience from the complete hardware implementation of phase 1 , we believe this estimate is fairly accurate.

The ECM unit including the full support for the phase 1 and 2 of the ECM with the word width $w=32$ bits, number of words $e=7$, level of pipeline $p=2$ has the following area requirements: 1754 LUTs, 506 flip-flops and 44 Blocks RAM. Minimum clock period is 26.225ns (maximum clock frequency: $38.132 \mathrm{MHz}$ ). Further improvements in data organization inside the ECM unit should yield higher performance of the whole design.

Due to the system's latency for loading and storing values in the registers, not more than 100 ECM units (FPGA) should be controlled by one processor. With a much higher number of units the communication overhead would outweigh the computation time. However, the control logic of the data I/O has not been in the focus of our optimization efforts yet and, thus, we assume that slight improvements of the speed of the data I/O are still feasible. Especially if targeting an ASIC implementation, such numbers are likely to change.

\section{A Case Study: Supporting GNFS with ECM ASICs}

Building an efficient and cheap ECM hardware can influence the overall performance of the GNFS since ECM can be perfectly used for smoothness testing within the GNFS (see [5]). In this section, we briefly estimate the costs, space requirements and power consumption of a special ECM hardware as ASIC. This special hardware could be produced as single ICs (such as common CPUs), ready for the use in larger circuits. We choose a setting with a word width $w=8$ and assume the use of carry save adders to allow for a higher clock rate (see [9]). 


\subsection{Estimation of the Running Time}

We can determine the running time of both phases on basis of the underlying ring arithmetic. The upper bounds for the number of clock cycles of a modular addition and a modular subtraction are given in Equations (5) and (6), respectively. A setting with $n=199, w=8, p=8$, and $e=25$ yields $T_{a d d}=3(e+1)=78$ and $T_{\text {sub }}=2(e+1)=52$ cycles. According to 4 , the implemented multiplier requires $T_{m u l}=666$ cycles. For each operation we should include $T_{\text {init }}=2$ cycles for initialization of ALU at the beginning of each computation.

For the group operations for phase 1 we obtain

$$
\begin{aligned}
& T_{\text {Padd }}=5 T_{\text {mul }}+3 T_{a d d}+3 T_{\text {sub }}+11 T_{\text {init }}=3742 \text { cycles and } \\
& T_{\text {Pdbl }}=5 T_{\text {mul }}+2 T_{a d d}+2 T_{\text {sub }}+9 T_{\text {init }}=3608 \text { cycles }
\end{aligned}
$$

clock cycles. For phase $2, T_{P a d d}$ changes to $T_{P a d d}^{\prime}=4410$ cycles since $z_{A-B} \neq 1$ in most cases, hence, we have to take the multiplication with $z_{A-B}$ into account.

The total cycle count for both phases is

$$
\begin{aligned}
& T_{\text {Phase 1 }}=1374\left(T_{\text {Padd }}+T_{\text {Pdbl }}\right)=10098900 \text { cycles and } \\
& T_{\text {Phase 2 }}=1881 T_{\text {Padd }}^{\prime}+50 T_{\text {Pdbl }}+13590 T_{\text {mul }}=17553730 \mathrm{cycles}
\end{aligned}
$$

clock cycles. Excluding the time for pre- and post-processing, a unit needs approximately $27.7 \times 10^{6}$ clock cycles for both phases on one curve. If we assume a frequency of $500 \mathrm{MHz}$, such a complex computation can be performed in approximately $55 \mathrm{~ms}$.

\subsection{Estimation of Area Requirements}

According to $[9]^{2}$, the multiplier with $w=8, p=8$ requires 21400 transistors in standard CMOS technology (assuming 4 transistors per NAND gate). We assume that the circuit for addition and subtraction can be achieved with at most 1000 transistors. For the memory, we assume (area expensive) static RAM which requires 25.200 transistors for 21 registers. For the control inside the unit and the control of the whole circuit, we assume additional 8.000 transistors per ECM unit.

\footnotetext{
${ }^{2}$ Remark: The numbers provided in that contribution refer to a multiplier built with carry save adders. Since we implemented the architecture with carry propagate adders, given numbers are larger (approximately 20\%) than those which would be achieved with our design.
} 
Hence, one unit requires approximately 55.600 transistors. Assuming the CMOS technology of a standard Pentium 4 processor $(0.13 \mu \mathrm{m}$, approx. Mio. 55 transistors), we could fit 990 ECM units into the area of one standard processor. One ECM unit needs an area of approximately $0.1475 \mathrm{~mm}^{2}$ and has a power dissipation of approximately $40 \mathrm{~mW}$.

\subsection{Application to the GNFS}

Considering the architecture for a special GNFS hardware of [5], we have to test approximately $1.7 \times 10^{14}$ rests up to 125 bits for smoothness. Since both the running time as well as the area requirement scales linearly with the bit size, we can multiply the results from the subsections above with a factor of $125 / 198 \approx 0.628$. If we distribute the computation over a whole year, we have to check 5.390.665 rests per second.

For a probability of success of $>90 \%$, we test 20 curves per rest, thus, we need approximately 3.850.000 ECM units which would yield a total chip area of $625.000 \mathrm{~mm}^{2}(=4300$ ICs of the size of a Pentium 4) and a power consumption of approximately $175 \mathrm{~kW}$. If we assume a cost of $\$ 5.000$ per $300 \mathrm{~mm}$ wafer, as done in [6], the ECM units would cost less than $\$ 45.000$ for the whole GNFS architecture, which is neglectable in the context of the overall costs.

\section{Conclusions}

With the work on hand, we present a thorough analysis of adequate algorithms for an ECM hardware architecture. The parameterization of the algorithms was done to particularly fit the needs of a hardware environment, yielding a high efficiency regarding the area-time product. Furthermore, this is the first publication showing a real hardware implementation of the ECM algorithm. The implementation is a hardware-software Co-design and has been implemented on an FPGA and an ARM microcontroller for factoring integers of a size of up to 198 bits. We implemented a variant of ECM which allows for a very low area time product and, hence, is very cost effective. Our implementation impressively shows that due to very low area requirements and low data I/O, ECM is predestined for the use in hardware. A single unit for factoring composites of up to 198 bits requires 506 flip-flops, 1754 lookup-tables and 44 BlockRAMs (less than $6 \%$ of logic and $27 \%$ of memory resources of the Xilinx Vertex2000E device). Regarding a possible ASIC implementation, around 990 ECM units could be placed on a single Pentium4-sized chip. 
As demonstrated, ECM can be perfectly parallelized and, thus, an implementation at a larger scale can be used to assist the GNFS factoring algorithm by carrying out all required smoothness tests. A low cost ASIC implementation of ECM can decrease the overall costs of the GNFS architecture SHARK, as shown in [5]. We believe that an extensive use of ECM for smoothness testing can further reduce the costs of such a GNFS machine.

As future steps, the control logic for the second phase will be finalized. Variants of phase 2 can be examined in order to achieve the lowest possible $A T$ product. To achieve a higher maximal clock frequency of the ECM unit, the control logic inside the unit might be optimized.

Since most of the computation time is spent for modular multiplications, an improvement of the implementation of the multiplication directly affects the overall performance. Hence, alternative architectures for the multiplication can be investigated.

With the VHDL source code at hand, the next logic step is the design and simulation of a full custom ASIC containing the logic, which currently is implemented in the FPGA. For an ASIC implementation, a parallel design of many ECM units is preferable. The control can still be handled outside the ASIC by a small microcontroller, as it is the case with the work at hand. Alternatively, a soft core of a small microcontroller can be adopted to the specific needs of ECM and be implemented within the ASIC. With a running ECM ASIC, exact cost estimates for the support of algorithms such as the GNFS can be obtained.

\section{References}

[1] R. L. Rivest, A. Shamir, and L. Adleman, "A Method for Obtaining Digital Signatures and Public-Key Cryptosystems," Communications of the ACM, vol. 21, pp. 120-126, February 1978.

[2] H. W. Lenstra, "Factoring Integers with Elliptic Curves," Annals of Mathematics, vol. 126, no. 2, pp. 649-673, 1987.

[3] A. K. Lenstra and H. W. Lenstra, The Development of the Number Field Sieve. Lecture Notes in Math. Volume 1554, 1993. 
[4] J. Franke, T. Kleinjung, F. Bahr, M. Lochter, and M. Bohm, "E-mail announcement." http://www.crypto-world.com/announcements/rsa576.txt, December 2003.

[5] J. Franke, T. Kleinjung, C. Paar, J. Pelzl, C. Priplata, and C. Stahlke, "SHARK — A Realizable Special Hardware Sieving Device for Factoring 1024-bit Integers." Submitted to SHARCS 2005 - Special-purpose Hardware for Attacking Cryptographic Systems, December 2004.

[6] A. Shamir and E. Tromer, "Factoring Large Numbers with the TWIRL Device," in Advances in Cryptology - Crypto 2003, vol. 2729 of LNCS, pp. 1-26, Springer, 2003.

[7] G. Orlando and C. Paar, "A Scalable $G F(p)$ Elliptic Curve Processor Architecture for Programmable Hardware," in Workshop on Cryptographic Hardware and Embedded Systems CHES 2001 (Ç. K. Koç, D. Naccache, and C. Paar, eds.), vol. LNCS 2162, pp. 348-363, Springer-Verlag, May 14-16, 2001.

[8] N. Gura, S. Chang, H. 2, G. Sumit, V. Gupta, D. Finchelstein, E. Goupy, and D. Stebila, "An End-to-End Systems Approach to Elliptic Curve Cryptography," in Cryptographic Hardware and Embedded Systems - CHES 2002 (Ç. K. Koç and C. Paar, eds.), vol. LNCS 2523, pp. 349-365, Springer-Verlag, 2002.

[9] A. Tenca and Ç.K. Koç, "A Scalable Architecture for Modular Multiplication Based on Montgomery's Algorithm," IEEE Trans. Comput., vol. 52, no. 9, pp. 1215-1221, 2003.

[10] D. Bernstein, "Circuits for Integer Factorization: A Proposal." Manuscript. Available at http://cr.yp.to/papers.html\#nfscircuit, 2001.

[11] E. Wolski, J. G. S. Filho, and M. A. R. Dantas, "Parallel Implementation of Elliptic Curve Method for Integer Factorization Using Message-Passing Interface (MPI)," in $S B A C$ - $P A D$ 13th Symposium on Computer Architecture and High-Performance, 2001, Pirenopolis - GO. SBAC-PAD 13th Symposium on Computer Architecture and High-Performance, 2001.

[12] P. Zimmermann, "ECMNET page." Available at http://www.loria.fr/ zimmerma/records/ecmnet.html.

[13] J. Pollard, "A Monte Carlo Method for Factorization," Nordisk Tidskrift for Informationsbehandlung (BIT), vol. 15, pp. 331-334, 1975. 
[14] R. P. Brent, "Factorization of the tenth Fermat number," Mathematics of Computation, vol. 68 , no. 225 , pp. 429-451, 1999.

[15] P. Montgomery, "Speeding up the Pollard and elliptic curve methods of factorization," Mathematics of Computation, vol. 48, pp. 243-264, 1987.

[16] R. P. Brent, "Some Integer Factorization Algorithms Using Elliptic Curves," in Australian Computer Science Communications 8, pp. 149-163, 1986.

[17] P. Montgomery, "Modular Multiplication without Trial Division," Mathematics of Computation, vol. 44, pp. 519-521, April 1985.

[18] M. Drutarovský, V. Fischer, and M. Šimka, "Comparison of two implementations of scalable Montgomery coprocessor embedded in reconfigurable hardware," in Proceedings of the XIX Conference on Design of Circuits and Integrated Systems - DCIS 2004, (Bordeaux, France), pp. 240-245, Nov. 24-26, 2004.

[19] NEC Corporation, "Preliminary User's Manual System-on-Chip Lite, Development Board, Hardware, Document No. A15650EE1V0UM00," July 2001.

Available at http://www.ee.nec.de/_pdf/A15650EE1VOUM00.PDF.

[20] Xilinx, "Virtex-E 1.8V Field Programmable Gate Arrays — Production Product Specification." http://www.xilinx.com/bvdocs/publications/ds022.pdf, June 2004.

[21] ARM Limited, "ARM7TDMI (Rev 3) — Technical Reference Manual.” Available at http://www.arm.com/pdfs/DDI0029G_7TDMI_R3_trm.pdf, 2001.

[22] A. O. L. Atkin and F. Morain, "Finding suitable curves for the elliptic curve method of factorization," Mathematics of Computation, vol. 60, no. 201, pp. 399-405, 1993. 


\section{A Appendix}

\section{A.1 Pollard's $(p-1)$-algorithm}

Let $k, N \in \mathbb{N}$ with $N$ being the composite to be factored. Furthermore, $q \mid N$ with $q \in \mathbb{P}$. Let $a \in \mathbb{Z}$ be coprime to $N$, i.e., $\operatorname{gcd}(a, N)=1$. Let $e=k(q-1)$.

By little Fermat,

$$
\begin{aligned}
& a^{q-1} \equiv 1 \bmod q \Rightarrow \quad a^{k(q-1)} \equiv 1 \bmod q \\
& \Leftrightarrow \quad a^{e}-1 \equiv 0 \bmod q \\
& \Leftarrow \quad q \mid a^{e}-1 . \\
& q \mid N \quad \text { yields } \quad \operatorname{gcd}\left(a^{e}-1, N\right)>1 . \\
& \text { If } \quad a^{e} \not \equiv 1 \bmod N \quad \text { then } \quad 1<g g T\left(a^{e}-1, N\right)<N .
\end{aligned}
$$

In this case, we found a non-trivial divisor of $N$.

We cannot compute $e=k(q-1)$ since $q$ is unknown. Instead, we assume that $q-1$ can be decomposed into many small factors below a certain threshold $B_{1}$. The highest prime power dividing $q-1$ is denoted as $B_{2} . q-1$ is then called $\left(B_{1}, B_{2}\right)$-smooth. Now, choose $e$ such that

$$
e=\prod_{p_{i} \in \mathbb{P}, p_{i} \leq B_{1}} p_{i}^{e_{p_{i}}}, \quad e_{p_{i}}=\max \left\{q \in \mathbb{N}: p_{i}^{q} \leq B_{2}\right\} .
$$

Hopefully, the computation of $a^{e}$ with $d=g g T\left(a^{e}-1, N\right)$ yields a non-trivial factor $d$ of $N$.

Generally, Pollard's method can be defined as follows:

Let $G_{q}:=(\mathbb{Z} / q \mathbb{Z})^{\star}$ and $G_{N}:=(\mathbb{Z} / N \mathbb{Z})^{\star}$ be multiplicative groups and let $G_{N} \rightarrow G_{q}, a \rightarrow \bar{a}$ be the reduction modulo $q$. A factor of $N$ is found, if $a^{e} \not \equiv 1 \bmod N$ and $a^{e} \equiv 1 \bmod q$, i.e.,

$$
\begin{aligned}
& \forall k_{1} \in \mathbb{N}: \quad e \neq k_{1} \times o(a), \\
& \exists k_{2} \in \mathbb{N}: \quad e=k_{2} \times o(\bar{a})
\end{aligned}
$$

with $o$ being the order of an element. 


\section{A.2 Finding Suitable Curves in Montgomery Form}

Assume a curve of the form

$$
B y^{2}=x^{3}+A x^{2}+x \quad \text { with } \quad \operatorname{gcd}\left(\left(A^{2}-4\right) B, n\right)=1
$$

Such curves have a group order divisible by 4 .

To obtain an order divisible by 12 , choose $A$ and $B$ such, that

$$
\begin{gathered}
A=\frac{-3 a^{4}-6 a^{2}+1}{4 a^{3}} \quad, \quad B=\frac{\left(a^{2}-1\right)^{2}}{4 a^{3}} \\
\text { with } a=\frac{t^{2}-1}{t^{2}+3} .
\end{gathered}
$$

The point

$$
\left(x_{0}, y_{0}\right)=\left(\frac{3 a^{2}+1}{4 a}, \frac{\sqrt{3 a^{2}+1}}{4 a}\right)
$$

is on the curve, if $3 a^{2}+1=4\left(t^{4}+3\right) /\left(t^{2}+3\right)^{2}$ is a rational square, which can be obtained by $t^{2}=\left(u^{2}-12\right) / 4 u$ and $u^{3}-12 u$ being a rational square. It is also possible to find suitable curves with torsion groups of order of 16 (see $[22,15]$ ) but yield no noticeable performance increase for our application.

\section{A.3 Useful Propositions}

Following Lemma allows us to reduce the complexity by repeatedly multiplying a difference of two products instead of computing complex point operations each step:

Lemma 1 Let $q=a+b$ with $a$ and $b$ coprime (which is true for any prime $p$ ). Furthermore, let $q Q=A+B$ with $A=a Q$ and $B=b Q$, then $z_{q Q}=0 \bmod t$ for $\operatorname{gcd}\left(z_{Q}, n\right)=1$ if and only if

$$
x_{A} \times z_{B}-z_{A} \times x_{B} \equiv 0 \bmod t .
$$

\section{Proof}

1. Montgomery's point addition formula yields

$$
\begin{aligned}
t \mid z_{q Q} & \Leftrightarrow t \mid x_{A-B}\left[x_{A} \times z_{B}-z_{A} \times x_{B}\right]^{2} \\
& \Leftarrow t \mid\left(x_{A} \times z_{B}-z_{A} \times x_{B}\right) .
\end{aligned}
$$


2. if $z_{q Q} \equiv 0 \bmod t, q Q$ is the identity point on the elliptic curve over $\mathbb{F}_{t}$. Hence, $A=-B$, i.e. $A$ and $B$ are zero or

$$
x_{A} / z_{A} \equiv x_{B} / z_{B} \bmod t .
$$

$A=B=0$ yields $Q=0$, thus $t \mid z_{Q}$, which is a contradiction to the assumption of $\operatorname{gcd}\left(z_{Q}, n\right)=$

1. Then we have

$$
\begin{aligned}
x_{A} / z_{A} & \equiv x_{B} / z_{B} \bmod t \quad \text { and } \\
x_{A} \times z_{B} & \equiv z_{A} \times x_{B} \bmod t \quad \text { respectively. }
\end{aligned}
$$

\section{A.4 Use of Multiple ALUs}

In this subsection, we will discuss the influence of using more than one ALU on the execution time and area of both phases. We have to find a trade-off between lowering the overall execution time and increasing area. Obviously, a naive approach would be the parallel use of 10 ALUs instead of one to compute every multiplication in parallel. But most operations depend on previously made computations and are, thus, not completely parallizable. Even if some operations are parallizable, their results have to be stored for post-processing, hence, more registers are required.

Typical operations during phase 1 are the point operations point addition and point duplication. Table 3 shows the sequence of required computations for a point addition (with $z_{P}=1$, as implemented for phase 1). The operations for multiplication, addition and subtraction are denoted as mul, add and sub, whereas the output operand is the first argument and the two input arguments are at the second and last position. $T_{i}$ are temporary registers. We identify at most two independent multiplication operations in this operation, i.e., before starting with the multiplications 9 and 10, the results of the multiplications from steps 3 and 6 (and some additions and subtractions) have to be available.

Hence, at most two ALUs can significantly improve the execution time of phase 1 . Table 4 shows the parallelized sequence of the point addition for two ALUs. In step 6 we can see ALU 1 running idle for the time of a multiplication since the point addition algorithm cannot be optimal parallelized. If we neglect the time for addition and subtraction, we can reduce the execution time by $40 \%$. The number of temporary registers increases by $50 \%$ from 4 to 6 .

The Table 5 and 6 show the computations for a point duplication with 1 and 2 ALUs, respectively. Similar to point addition, we cannot manage an optimum parallization since ALU 1 is 
Table 3: Point Addition with 1 ALU (Pseudocode)

\begin{tabular}{|c|l|}
\hline Step & ALU 1 \\
\hline \hline 1 & $\operatorname{sub}\left(T_{1}, x_{P 1}, z_{P 1}\right)$ \\
2 & $\operatorname{add}\left(T_{2}, x_{P 2}, z_{P 2}\right)$ \\
3 & $\operatorname{mul}\left(T_{3}, T_{1}, T_{2}\right)$ \\
4 & $\operatorname{add}\left(T_{1}, x_{P 1}, z_{P 1}\right)$ \\
5 & $\operatorname{sub}\left(T_{2}, x_{P 2}, z_{P 2}\right)$ \\
6 & $\operatorname{mul}\left(T_{4}, T_{1}, T_{2}\right)$ \\
7 & $\operatorname{add}\left(T_{1}, T_{3}, T_{4}\right)$ \\
8 & $\operatorname{sub}\left(T_{2}, T_{3}, T_{4}\right)$ \\
9 & $\operatorname{mul}\left(x_{P 1+P 2}, T_{1}, T_{1}\right)$ \\
10 & $\operatorname{mul}\left(T_{3}, T_{2}, T_{2}\right)$ \\
11 & $\operatorname{mul}\left(z_{P 1+P 2}, x_{P 1-P 2}, T_{3}\right)$ \\
\hline \hline$\sum$ & $t_{s u m} \approx 6 \times t_{a d d}+5 \times t_{m u l}$ \\
\hline
\end{tabular}

Table 4: Point Addition with 2 ALUs (Pseudocode)

\begin{tabular}{|c|l|l|}
\hline Step & ALU 1 & ALU 2 \\
\hline \hline 1 & $\operatorname{sub}\left(T_{1}, x_{P 1}, z_{P 1}\right)$ & $\operatorname{add}\left(T_{4}, x_{P 1}, z_{P 1}\right)$ \\
2 & $\operatorname{add}\left(T_{2}, x_{P 2}, z_{P 2}\right)$ & $\operatorname{sub}\left(T_{5}, x_{P 2}, z_{P 2}\right)$ \\
3 & $\operatorname{mul}\left(T_{3}, T_{1}, T_{2}\right)$ & $\operatorname{mul}\left(T_{6}, T_{4}, T_{5}\right)$ \\
4 & $\operatorname{add}\left(T_{1}, T_{3}, T_{6}\right)$ & $\operatorname{sub}\left(T_{2}, T_{3}, T_{6}\right)$ \\
5 & $\operatorname{mul}\left(x_{P 1+P 2}, T_{1}, T_{1}\right)$ & $\operatorname{mul}\left(T_{3}, T_{2}, T_{2}\right)$ \\
6 & & $\operatorname{mul}\left(z_{P 1+P 2}, x_{P 1-P 2}, T_{3}\right)$ \\
\hline \hline$\sum$ & $t_{\text {sum }} \approx 3 \cdot\left(t_{\text {add }}+t_{\text {mul }}\right)$ & \\
\hline
\end{tabular}

running idle for some time.

The overall running time in the parallelized algorithm can be reduced by approximately $40 \%$, the number of required registers for intermediate results stays constant.

Phase 2, parameterized according to Section 4.1, is more complicated to parallelize. All point operations can be parallelized as described above. The computations for the product involve three multiplications, where two can be executed in parallel. Hence, an acceleration of approximately $35 \%$ can be achieved with two $\mathrm{ALUs}^{3}$.

Combining both phases, two ALUs yield a total speed up of $37 \%$. Hence, the AT product of two ALUs is higher than that of a single ALU. Additionally, two more registers for intermediate

\footnotetext{
${ }^{3}$ Remark: A slightly improved acceleration could be attained by investigating a higher degree of optimization by interleaving parts of the operations.
} 
Table 5: Point Duplication with 1 ALU (Pseudocode)

\begin{tabular}{|c|l|}
\hline Step & ALU 1 \\
\hline \hline 1 & $\operatorname{add}\left(T_{1}, x_{P}, z_{P}\right)$ \\
2 & $\operatorname{sub}\left(T_{2}, x_{P}, z_{P}\right)$ \\
3 & $\operatorname{mul}\left(T_{3}, T_{1}, T_{1}\right)$ \\
4 & $\operatorname{mul}\left(T_{4}, T_{2}, T_{2}\right)$ \\
5 & $\operatorname{sub}\left(T_{1}, T_{3}, T_{4}\right)$ \\
6 & $\operatorname{mul}\left(T_{2}, T_{1}, A_{24}\right)$ \\
7 & $\operatorname{mul}\left(x_{2 P}, T_{3}, T_{4}\right)$ \\
8 & $\operatorname{add}\left(T_{3}, T_{2}, T_{4}\right)$ \\
9 & $\operatorname{mul}\left(z_{2 P}, T_{1}, T_{3}\right)$ \\
\hline \hline$\sum$ & $t_{\text {sum }} \approx 4 \cdot t_{a d d}+5 \cdot t_{m u l}$ \\
\hline
\end{tabular}

Table 6: Point Duplication with 2 ALUs (Pseudocode)

\begin{tabular}{|c|l|l|}
\hline Step & ALU 1 & ALU 2 \\
\hline \hline 1 & $\operatorname{add}\left(T_{1}, x_{P}, z_{P}\right)$ & $\operatorname{sub}\left(T_{2}, x_{P}, z_{P}\right)$ \\
2 & $\operatorname{mul}\left(T_{3}, T_{1}, T_{1}\right)$ & $\operatorname{mul}\left(T_{4}, T_{2}, T_{2}\right)$ \\
3 & $\operatorname{sub}\left(T_{1}, T_{3}, T_{4}\right)$ & \\
4 & $\operatorname{mul}\left(T_{2}, T_{1}, A_{24}\right)$ & $\operatorname{mul}\left(x_{2 P}, T_{3}, T_{4}\right)$ \\
5 & $\operatorname{add}\left(T_{3}, T_{4}, T_{2}\right)$ & \\
6 & $\operatorname{mul}\left(z_{2 P}, T_{1}, T_{3}\right)$ & \\
\hline$\sum$ & $t_{\text {sum }} \approx 3 \cdot t_{\text {add }}+3 \cdot t_{\text {mul }}$ & \\
\hline
\end{tabular}

values are required. However, considering the AT complexity of the entire ECM unit, the AT product does slightly decrease. The reason for this behavior is the impact of the area due to the 21 registers of the unit. An additional ALU and 2 more registers only slightly influence the total area consumption, hence, yielding a lower AT product. 


\section{List of Figures}

1 Overview of one ECM Unit . . . . . . . . . . . . . . . . . . . . . . . 12

2 Memory Management of the ECM Unit . . . . . . . . . . . . . . . . . . . 13

3 Multiplier Stage with Carry-Propagate Adders and Non-redundant Representation 15

4 Addition and Subtraction Circuit . . . . . . . . . . . . . . . . . 16

$5 \quad$ Parallel ECM . . . . . . . . . . . . . . . . . . . . . . . . 18 